\title{
Towards Semantic Interoperability in an Open IoT Ecosystem for Connected Vehicle Services
}

\author{
Niklas Kolbe, Sylvain Kubler, Jérémy Robert, Yves Le Traon \\ Interdisciplinary Center for Security, Reliability and Trust \\ University of Luxembourg \\ Luxembourg, Luxembourg \\ \{niklas.kolbe, sylvain.kubler, jeremy.robert, yves.letraon\}@uni.lu
}

\author{
Arkady Zaslavsky \\ Data61 \\ CSIRO \\ Clayton, Australia \\ arkady.zaslavsky@csiro.au
}

\begin{abstract}
A present challenge in today's Internet of Things (IoT) ecosystem is to enable interoperability across heterogeneous systems and service providers. Restricted access to data sources and services limits the capabilities of a smart city to improve social, environmental and economic aspects. Interoperability in the IoT is concerned with both, messaging interfaces and semantic understanding of heterogeneous data. In this paper, the first building blocks of an emerging open IoT ecosystem developed at the EU level are presented. Semantic web technologies are applied to the existing messaging components to support and improve semantic interoperability. The approach is demonstrated with a proof-of-concept for connected vehicle services in a smart city setting.
\end{abstract}

Keywords-Semantic Web of Things, Interoperability, Smart Ecosystem, Connected Vehicle

\section{INTRODUCTION}

In the smart city concept, ICT facilitates the development of social, environmental, and economic services and solutions. It has been widely acknowledged that IoTenabled ecosystems have the potential to improve living conditions in cities regarding mobility, environment, governing, healthcare, safety, and so forth [1]. Furthermore, the access to overarching relevant information allows for the development of sustainable solutions regarding global challenges like climate change, food security, and resource depletion [2]. Vehicles connected to the infrastructure of a smart ecosystem, for example, enable services that could improve transportation, reduce environmental impact and improve the life quality of citizens [3].

In order to achieve these goals in a smart and open ecosystem, various types of information need to be made accessible and services from across different domains need to interact with each other. However, a critical issue remains to be solved, namely the vertical silos that shape today's Internet of Things (IoT) [4]. These silos are proprietary systems that evolve due to the different interests of involved stakeholders like users, developers, companies and public institutions. As a consequence, the opportunities that a smart city could offer, or the innovate potentials of an emergent business, are limited due to the restricted access to data sources and the non-standardized way of publishing and accessing IoT data/services. In this paper, IoT data refers to streams of generated data (e.g. from sensors), whereas IoT services refers to computed results based on requests with input parameters, potentially relying on IoT data.

In order to move towards a truly connected city, various interoperability-related challenges have to be addressed, e.g. regarding messaging, semantics and access policies. This paper focuses on semantic interoperability, providing a brief overview of how this issue is tackled in an ongoing H2020 project. The approach is applied to a connected vehicle use case scenario. The technical contribution of this paper includes the integration of linked vocabularies into a standardized data format for the IoT. Section II presents the context of this work and the necessary background regarding semantic interoperability. Section III presents relevant components of the open IoT ecosystem. Section IV describes the use case scenario and implementation; the conclusion follows.

\section{TOWARDS AN OPEN IOT ECOSYSTEM}

Establishing an open IoT ecosystem, e.g. for smart cities, requires a joint effort of administrative, academic and industrial institutions. In the following the EU initiatives and relevant background for semantic interoperability in the IoT is presented.

\section{A. EU Vision and Initiatives}

The IoT ambition of the EU is a network of open IoT ecosystems in which small and medium-sized enterprises are able to participate in offering smart services on the market. Ongoing efforts are built upon existing IoT platforms which are outcomes of past EU initiatives [5]. In order to move from research and innovation towards market deployment, the ongoing H2020 IoT programmes aim to foster innovation ecosystems, advancing in IoT standardisation and interoperability, as well as deploying large-scale pilots.

There are currently seven H2020 Research and Innovation IoT-EPI projects that aim to improve horizontal interoperability between smart objects, as presented in detail in [6]. Eventually, this IoT convergence would form an open IoT 
ecosystem in which services in any scale could be offered, traded and monetized on the market.

\section{B. Semantic Interoperability in the IoT}

Semantic interoperability for the IoT refers to achieving a general semantic understanding of the communication between things. However, interoperability in the IoT concerns various layers of the communication stack. The advancements of integrating smart things in the various layers are labelled with Internet of Things (network layer), Web of Things (application layer) and Semantic Web of Things (a common description of things) [7]. Since the underlying layers adopted standard technologies of the web (such as HTTP/HTML), leveraging semantic web technologies for the IoT is a promising approach to converge heterogeneous data sources in a smart ecosystem [8].

The Resource Description Format (RDF) [9] is the major technology supporting the movement to a Semantic Web of Things. RDF allows to describe and reference entities and their relationships via URIs in a structured form. Linked Data [10], for example, is based on RDF and allow for defining, linking and querying concepts. The main motivation to move towards the Semantic Web of Things is to achieve a common understanding of the labels which are annotated to the data that is exchanged.

To achieve this universal understanding of messages, all participants of the communication must be able to interpret the used vocabulary. RDF provides a flexible approach to support and specify domain-dependent and independent vocabularies, and to define relations between terms from different vocabularies [7]. However, even though the vocabulary can be easily accessed, consumers must be able to understand the used vocabulary by the publisher, by either using the same or an aligned vocabulary. This is a known issue in the semantic web community [11] and is also being investigated for the Semantic Web of Things.

Linked Open Vocabularies (LOV) [12], for example, is a RDF-based repository in which data publishers and consumers can lookup vocabularies that could be used to annotate and parse messages. Several initiatives aiming at defining domain-dependent and independent vocabularies can be found, such as schema.org ${ }^{1}$. MobiVoc ${ }^{2}$, for example, is an initiative for a domain-dependent RDF vocabulary for mobility. Additionally, high-level conceptualizations like the Semantic Sensor Network ontology $(\mathrm{SSN})^{3}$ provide further semantic specifications of things and their relations. In SSN, for instance, sensors, their setup, and their observations can be defined, which in turn could be combined with other vocabularies that define labels for specific sensor types, observations, attributes, and so forth.

\footnotetext{
${ }^{1}$ Schema.org vocabulary: http://schema.org/

${ }^{2}$ MobiVoc vocabulary: http://schema.mobivoc.org/

${ }^{3}$ SSN ontology: https://www.w3.org/2005/Incubator/ssn/ssnx/ssn
}

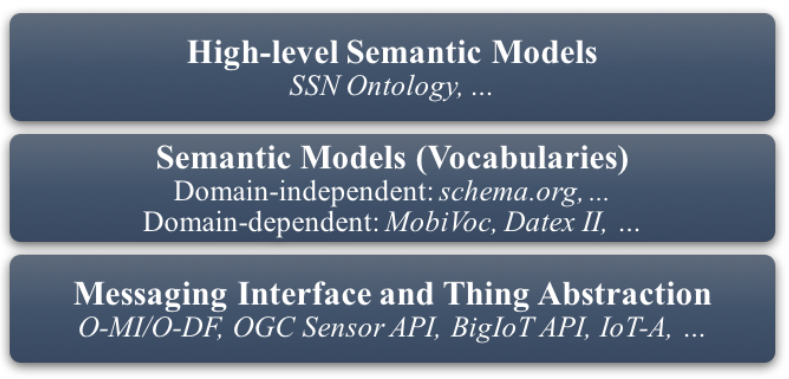

Figure 1. Positioning of Semantic Techniques in IoT Communication Stack, inspired by [7], [8]

Figure 1 illustrates how these semantic models relate to existing messaging interfaces and thing description models in the IoT. Semantic models are not restricted to RDF, DATEX $\mathrm{II}^{4}$ for example, is a EU initiative to define a vocabulary for the transportation domain in UML and the data models of the FIWARE ${ }^{5}$ initiative are represented in JSON. The layer below the semantic models is responsible for the definition of messaging interfaces and supporting the abstraction of smart things. Past and ongoing initiatives for this layer are, for example, IoT-A ${ }^{6}$, the OGC SensorThings $\mathrm{API}^{7}$, and the BIGIoT API [13].

The open messaging standards called Open-Messaging Interface $(\mathrm{O}-\mathrm{MI})^{8}$ and Open-Data Format $(\mathrm{O}-\mathrm{DF})^{9}$ are used in the open ecosystem to achieve interoperability on the application layer. These standards will be discussed in more detail in the following section. The proof-of-concept of this paper combines an RDF vocabulary for the mobility domain (MobiVoc) with O-MI/O-DF to benefit the development of connected vehicle services in a smart ecosystem.

\section{BIOTOPE ECOSYSTEM FOUNDATION}

The work presented in the following is part of the bIoTope project $^{10}$. The building blocks of the bIoTope ecosystem, as illustrated in Figure 2, are (i) O-MI, (ii) O-DF, (iii) data/service publishers and (iv) consumers, as well as (v) a service description repository. Furthermore, the figure presents the typical interactions between these components and technologies.

The bIoTope ecosystem relies on the O-MI standard (i) as a messaging interface between data/service publishers/consumers in the IoT. An agent is responsible to push the data from any kind of source (sensor network gateways, APIs, local files, databases, UI etc.) to an O-MI node. The interface

\footnotetext{
${ }^{4}$ DATEX II model: http://www.datex2.eu/

${ }^{5}$ FIWARE models: https://www.fiware.org/data-models/

${ }^{6}$ IoT-A project: http://www.iot-a.eu/

${ }^{7}$ OGC SensorThings API: https://github.com/opengeospatial/sensorthings

${ }^{8} \mathrm{O}-\mathrm{MI}$ standard: https://www2.opengroup.org/ogsys/catalog/C14B

${ }^{9} \mathrm{O}-\mathrm{DF}$ standard: https://www2.opengroup.org/ogsys/catalog/C14A

${ }^{10}$ bIoTope project: http://biotope-project.eu/
} 


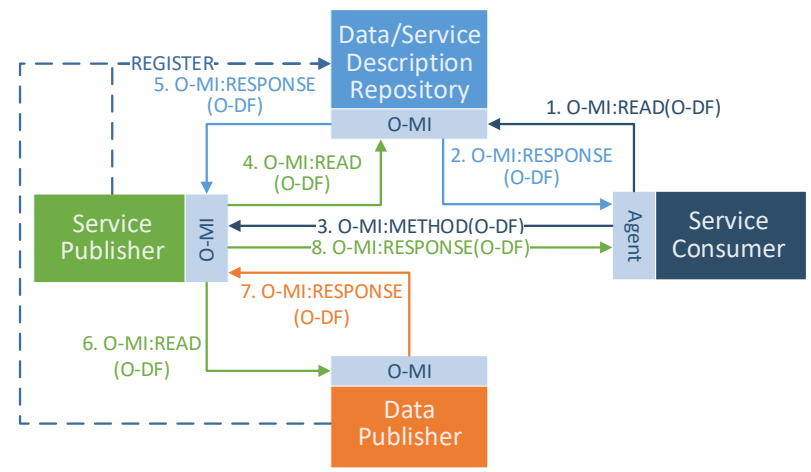

Figure 2. Publish-Find-Bind Pattern applied to the bIoTope Ecosystem

includes operations to read, write and subscribe to values, as well as invoking methods with parameters.

The O-DF standard (ii) defines the structure of the message payload. O-DF provides a tree structure of objects which are comprised of infoitems with values and meta-data. An example of O-MI/O-DF messaging will be presented in section IV. Both standards have been thoroughly discussed and designed to meet the requirements of all involved stakeholders in an IoT ecosystem as presented in [14], [15].

Publishers (iii) can be differentiated in those exposing data and those offering more complex services. Data publishers in the bIoTope ecosystem refer to exposing low-level data via the O-MI operations read, write and subscribe. Service publishers compose high-level services by gathering lowlevel data, processing it, and exposing the results via the O-MI operation method. Consumers (iv) are calling service methods or reading low-level data to compose smart services for the ecosystem.

The service description repository (v) provides an index of available O-MI nodes and their exposed O-DF tree. Publishers register their services in the repository and consumers use the repository as an entry point to discover relevant services. The repository provides an O-MI interface that allows to search and filter available O-MI nodes and returns the data required to access the matching services in a peer-to-peer manner.

O-DF was designed to provide a general message format without restricting involved actors in their width of information that can be exchanged. Thus, the goal is to achieve semantic interoperability between the presented consumers, publishers and the service description repository on top of O-DF payload.

\section{Use CASE DESCRIPTION}

For demonstration purposes of the presented smart ecosystem components, it is assumed that vehicles are connected to the internet. The vehicles expose their data (location, car profile, etc.) to a back end server. Moreover, the vehicle is capable of receiving remotely computed information and is able to integrate the data into the services that are offered to the driver in the connected vehicle.

The scenario involves the following implementations: A back end agent that acts as a gateway to the connected vehicles and as a service consumer in the ecosystem, three service publishers (find parking, find charging station and predict free parking), one data publisher (real time parking data), and the service description repository.

The Lyon Greater Region is the considered use case setting. It will be demonstrated how the consumer is able to dynamically discover and request relevant services, as well as to further process the received results, due to the usage of a standardized vocabulary. Figure 3 shows an instantiated execution flow of this scenario involving the find parking service.

The data publishing component is realised by accessing real time parking data via the open data platform of Lyon ${ }^{11}$. The agent requests the parking data from the open data platform API every minute and transforms the received JSON object into an MobiVoc annotated O-DF tree, which is then pushed to the O-MI node. A fragment of the O-DF tree is shown in Listing 1 that integrates terms of the MobiVoc and schema.org vocabulary.

\section{Listing 1}

O-DF Tree with Semantic Annotations For Parking Data

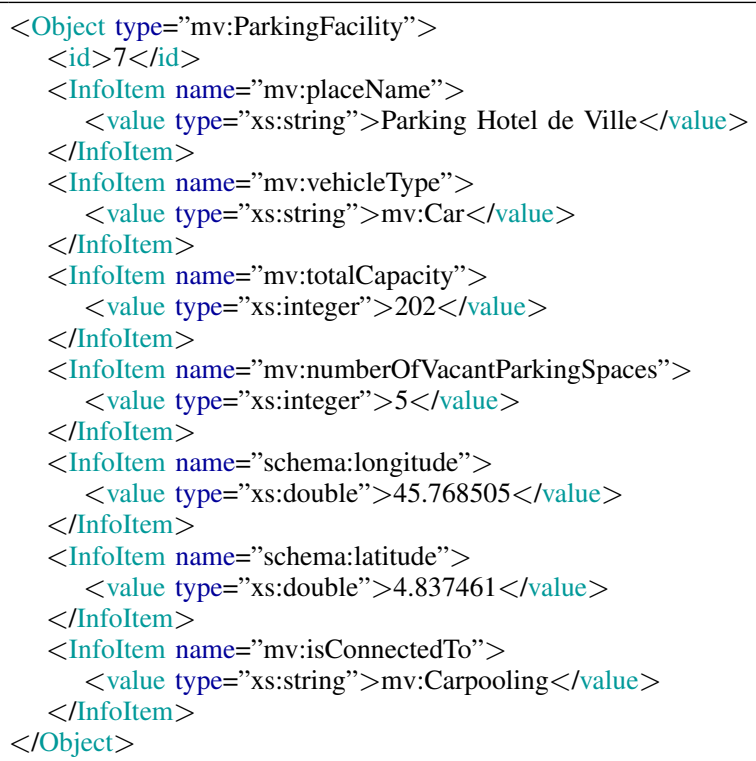

The service publishers are realised as individual implementations based on the flow that was presented in Figure 2. Upon a method call, service publishers request the service descriptions from the repository by using terms from the MobiVoc vocabulary (and a geo-filter) to discover relevant service and data publishers. The service descriptions are used to establish peer-to-peer connections to these nodes.

\footnotetext{
${ }^{11}$ Data Grand Lyon: https://data.grandlyon.com/
} 


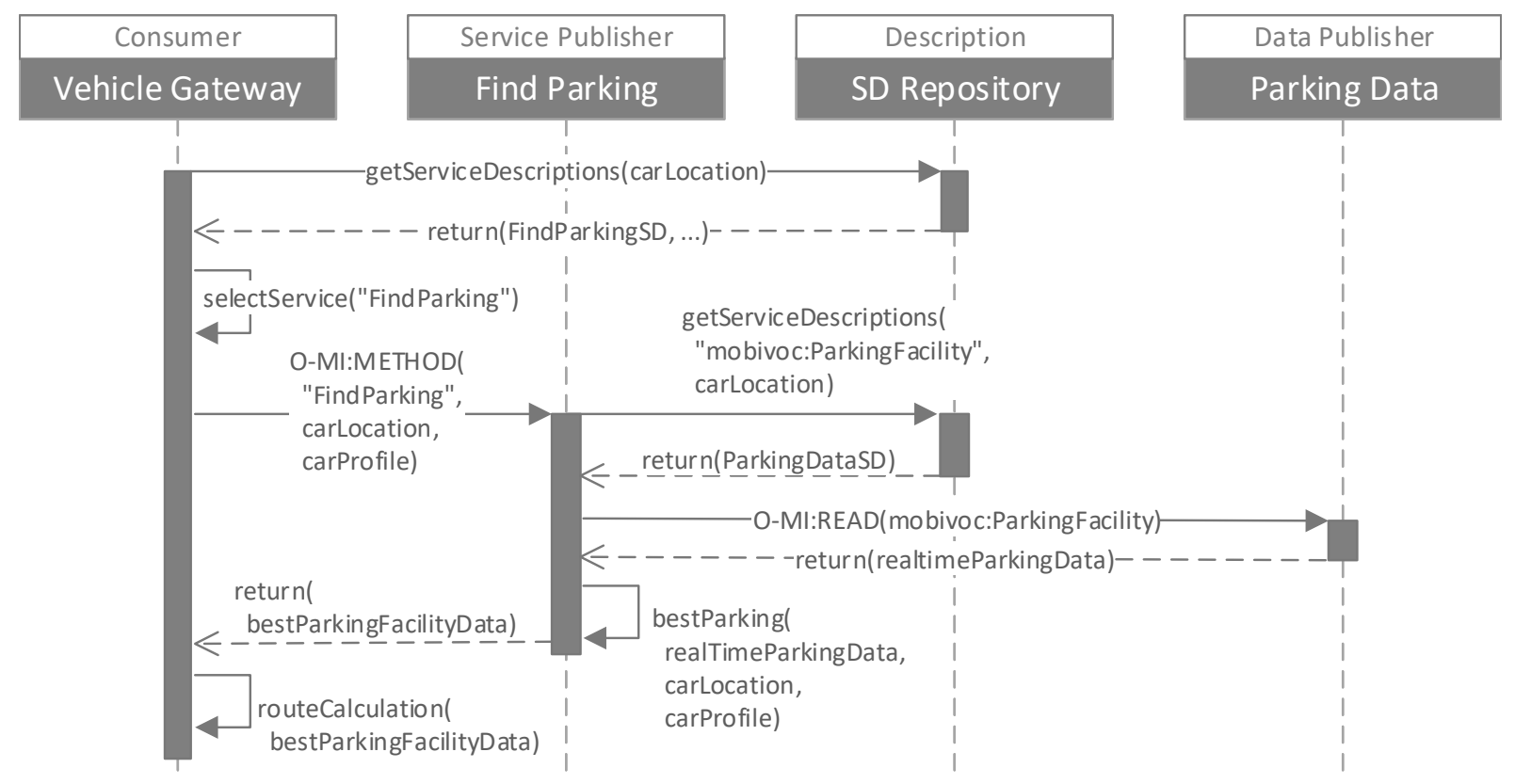

Figure 3. Sequence Diagram for Smart Parking Scenario

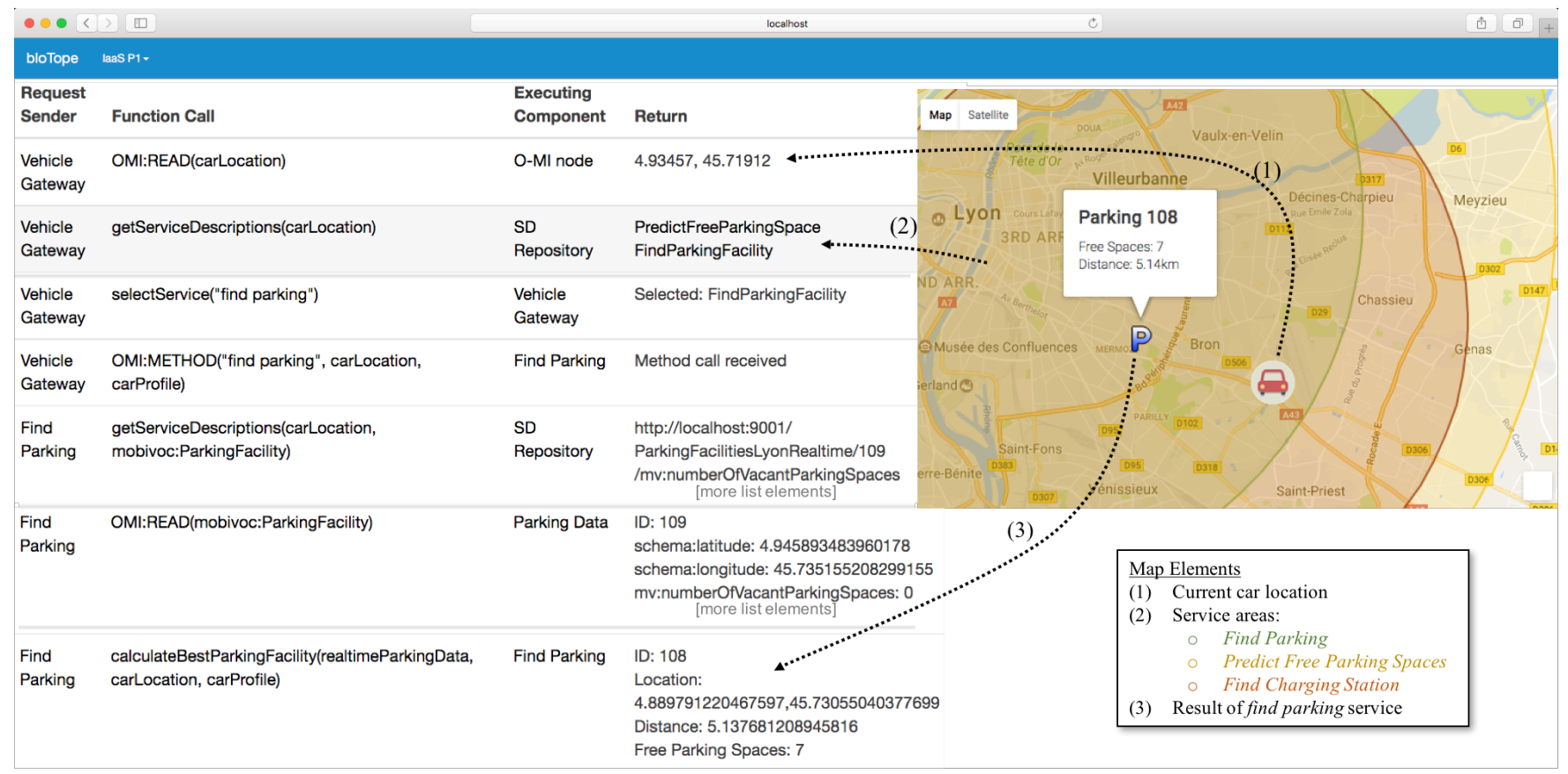

Figure 4. Proof-of-concept UI with Annotations to observe Function Calls and Results for Lyon Use Case

The collected data is used to calculate the results of the service implementation and to be sent as the response to the consumer. In case of the find parking service, the MobiVoc term ParkingFacility is used to discover publishers with parking information. The service algorithm takes the numberOfVacantParkingSpaces, the location and profile of the vehicle, as well as the location of the parking facilities into account to send the data of the best parking facility as response to the initial request of the vehicle gateway.

All publishers are indexed in the service description repository with respective URLs and other meta-data taken from the O-DF tree. Furthermore, service publishers are 
assigned with a geographic service area in which the service is valid. Upon service description requests with a location parameter, only service descriptions for services that are assigned for this location are returned.

A web interface is used to monitor the execution flow and visualise the service results, as shown in Figure 4. The location of the vehicles are simulated by an agent that updates the values every second via an O-MI node. The execution flow is initiated by the vehicle gateway by reading the car location and initiating the service discovery, selection and execution process.

The eventual result of the find parking service contains the semantic annotated data for the best parking facility. In the described scenario, this data is used by the vehicle gateway to calculate a route to the location of this parking facility. The route is sent to the vehicle and displayed to the driver via the vehicle's user interface.

\section{CONCLUSION}

This paper presented ongoing initiatives for open IoT ecosystems and challenges for semantic interoperability. The integration of semantic web technologies into the existing building blocks of the bIoTope project was discussed to approach the aforementioned challenges. A proof-of-concept demonstrated the application of this approach for a parking scenario in a smart city setting with connected vehicles.

Future work is concerned with the extension of the implementations for the large-scale city use cases and pilots. This includes further integration of contextual data in the ecosystem, fully harnessing the potential of semantic technologies and developing advanced services, as for example parking reservations, billing services, and conflict resolutions for competing cars.

\section{ACKNOWLEDGMENT}

The research leading to this publication is supported by the EUs H2020 Programme for research, technological development and demonstration (grant 688203). Authors acknowledge financial support from Ministry of Science \& Education of Russian Federation, Grant RFMEFI58716X0031.

\section{REFERENCES}

[1] A. Zanella, N. Bui, A. Castellani, L. Vangelista, and M. Zorzi, "Internet of things for smart cities," IEEE Internet of Things journal, vol. 1, no. 1, pp. 22-32, 2014.

[2] P. Neirotti, A. De Marco, A. C. Cagliano, G. Mangano, and F. Scorrano, "Current trends in smart city initiatives: Some stylised facts," Cities, vol. 38, pp. 25-36, 2014.

[3] W. He, G. Yan, and L. Da Xu, "Developing vehicular data cloud services in the iot environment," IEEE Transactions on Industrial Informatics, vol. 10, no. 2, pp. 1587-1595, 2014.

[4] O. Vermesan and P. Friess, "Digitising the industry-internet of things connecting the physical, digital and virtual worlds," 2016.
[5] - Internet of Things-From Research and Innovation to Market Deployment. River Publishers Aalborg, 2014.

[6] S. Kubler, J. Robert, A. Hefnawy, C. Cherifi, A. Bouras, and K. Främling, "Iot-based smart parking system for sporting event management," in Proceedings of the 13th International Conference on Mobile and Ubiquitous Systems: Computing, Networking and Services. ACM, 2016, pp. 104-114.

[7] A. J. Jara, A. C. Olivieri, Y. Bocchi, M. Jung, W. Kastner, and A. F. Skarmeta, "Semantic web of things: an analysis of the application semantics for the iot moving towards the iot convergence," International Journal of Web and Grid Services, vol. 10, no. 2-3, pp. 244-272, 2014.

[8] P. Barnaghi, W. Wang, C. Henson, and K. Taylor, "Semantics for the internet of things: early progress and back to the future," International Journal on Semantic Web and Information Systems (IJSWIS), vol. 8, no. 1, pp. 1-21, 2012.

[9] D. Brickley and R. V. Guha, "Rdf vocabulary description language 1.0: Rdf schema," 2004.

[10] T. Heath and C. Bizer, "Linked data: Evolving the web into a global data space," Synthesis lectures on the semantic web: theory and technology, vol. 1, no. 1, pp. 1-136, 2011.

[11] J. Euzenat, "An api for ontology alignment," in International Semantic Web Conference. Springer, 2004, pp. 698-712.

[12] P.-Y. Vandenbussche, G. A. Atemezing, M. Poveda-Villalón, and B. Vatant, "Linked open vocabularies (lov): a gateway to reusable semantic vocabularies on the web," Semantic Web, vol. 8, no. 3, pp. 437-452, 2017.

[13] A. Bröring, S. Schmid, C.-K. Schindhelm, A. Khelil, S. Kaebisch, D. Kramer, D. Le Phuoc, J. Mitic, D. Anicic, E. Teniente et al., "Enabling iot ecosystems through platform interoperability," IEEE Software, 2017.

[14] K. Främling, S. Kubler, and A. Buda, "Universal messaging standards for the iot from a lifecycle management perspective," IEEE Internet of things journal, vol. 1, no. 4, pp. 319327, 2014.

[15] S. Kubler, M. Madhikermi, A. Buda, and K. Främling, "Qlm messaging standards: introduction and comparison with existing messaging protocols," in Service Orientation in Holonic and Multi-Agent Manufacturing and Robotics. Springer, 2014, pp. 237-256. 